that is :

$$
R_{n}=\frac{r_{n} t}{t_{n}}=\frac{r_{0}+\alpha t_{n}}{t_{n}} \cdot t=\left(\frac{r_{0}}{t_{n}}+\alpha\right) t
$$

where $R_{n}$ is the actual orbital radius, $r_{0}$ the orbital radius at the moment of formation, that is, at the time $t_{n}$.

The graph indicates that the value $t_{n}$ is-at least within a certain time interval-roughly twice the preceding value, so that:

$$
R_{n}=\left(\frac{r_{0}}{2^{n} t_{0}}+\alpha\right) t=\frac{C}{2^{n}}+D
$$

in agreement with the 'law' of Titius and Bode.

The masses escaping from the Sun will invariably be those of highest specific impulse momentum. Thus it can be understood why the bulk of the impulse momentum of the solar system is borne by the planets.

Gravity differentiation in the Sun must have been most intense at the time when gravitational acceleration was greatest. Therefore, the older, that is, the outer, planets are of smaller bulk density.

The above mechanism can be extended to the satellites of the larger planets, too. The coincidence of the orbital planes is a matter-of-fact corollary and the formation of rings or asteroids is also possible.

This theory is obviously far from being complete. I wish merely to show that the results obtained by Dirac and by Gilbert may in themselves be the nucleus of a theory of the origin of the solar system.

Geophysical Institute, Eötvös University, Budapest.

1 Dirac, P. A. M., Proc. Roy. Soc., A, 165, 199 (1938). ${ }^{2}$ Gilbert, C., Mon. Not. Roy. Astro. Soc., 116, 684 (1956).

\section{The Extragalactic Distance Scale}

THE establishment of the scale of distances in the extragalactic universe rests primarily on the determinations of the distances of the nearest galaxies. These distances have been obtained primarily from a comparison of apparent magnitudes of variable stars or of Population II giants with the known absolute magnitudes of such objects measured in our Galaxy.

Arp $^{1}$ has obtained colour-magnitude diagrams for four clusters in the Small Magellanic Cloud and has found that these diagrams are different from colour-magnitude diagrams of similar objects in the Galaxy. Using a faint sequence of magnitudes lent to me by Arp, I have obtained colour-magnitude diagrams for six clusters of the Large Magellanic Cloud and have found that the diagrams differ not only from those found in the Galaxy but also from those found by Arp in the Small Magellanic Cloud. Three of those studied by me are globular clusters : $N G C$ 1846, NGC 1978, and Anonymous 4. Their giant branches have slopes much more gentle than any observed in globular clusters of our Galaxy. Even the giant branch of $N G C 6356$, which Sandage and Wallerstein $^{2}$ find to have an unusually gentle slope, is steeper than those in the clusters of the Large Cloud.

$N G C 1831$ and $N G C 1818$, two of the three other clusters, are similar in appearance to globular clusters, but are much younger, with colour-magnitude diagrams somewhat similar to those of $M 11^{3}$ and $h$ and $\chi$ Persei ${ }^{4}$, respectively. They differ from open clusters of our Galaxy not only in their much larger numbers of stars but also in the unexpected location of the giants in their colour-magnitude diagrams. The sixth cluster, $N G C$ 1844, a small open cluster, shares this latter peculiarity. NGC 1844 has a main sequence similar to that of $N G C 4755^{5}$, but its giant stars are bluer and brighter.

Because the distances to the nearby galaxies are determined from the apparent magnitudes of the giant evolved stars such as the Cepheids, the $R R$ Lyrae variables, and the brightest stars of Population II, the evidence presented by the Magellanic Clouds indicates that these distances are not necessarily correct. The characteristics of the giant stars, and their evolutionary paths, appear to differ in different galaxies and we may not safely use their apparent magnitudes as an index of distance until we thoroughly understand these differences.

Harvard College Observatory.

PaUL W. Hodge

$$
\text { April } 6 .
$$

'Arp, H. C., Astronom. J., 63, 273, 487 (1958); 64, 175, 254 (1959). "Sandage, A. R., and Wallerstein, G., Astrophys. J. (in the press). ${ }^{3}$ Johnson, H. L., and Hiltner, W. A., Astrophys. J., 128, 267 (1956). -Johnson, H. L., Sandage, A. R., and Wahlquist, H. D., Astrophye. J., 124, 81 (1956).

'Arp, H. C., and van Sant, C. T., Astronom. J., 63, 341 (1958).

\section{RADIOPHYSICS}

\section{Faraday Effect in the Transmissions from Fast Spinning Satellites}

The interaction of the satellite spin and the Faraday rotation effect in the ionosphere in producing polarization fading in linearly polarized signals from Earth satellites has been discussed for the case when the satellite rotation period is of the same order as the Faraday fading period ${ }^{1,2}$. In this case, determination of the rate of Faraday fading is difficult unless simultaneous fading records on two frequencies can be taken.

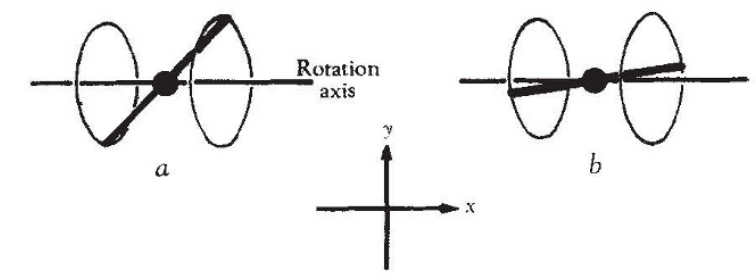

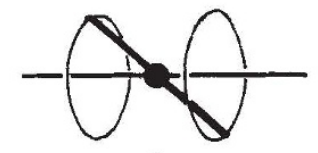

c
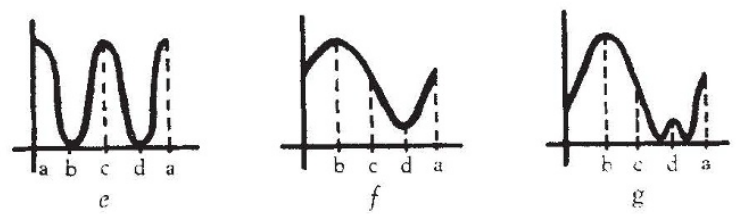

$g$
Fig. 1. Four positions of a dipole with its rotation axis and axis of symmetry not coincident. The corresponding signal strength
variations for polarizations in the $x$ and $y$ directions are shown variations for polarizations in the $x$ and $y$ directions are shown
at $f$ and $e$ when the line of sight is outside the generated cone, at $f$ and $e$ when the line of sight is outside the
and at $g$ and $e$ when it is inside 